\title{
PERFORMANCE ANALYSIS OF TURBO CODES OVER NAKAGAMI- $M$ FADING CHANNELS WITH IMPULSIVE NOISE
}

\author{
Syed Amjad Ali \\ Bilkent University \\ Ankara, Turkey
}

\section{ABSTRACT}

The statistical characteristics of impulsive noise differ greatly from those of Gaussian noise. Hence, the performance of conventional decoders, optimized for AWGN channels is not promising in non-Gaussian environments. In order to achieve improved performance in impulsive environments the decoder structure needs to be adapted in accordance with the impulsive noise model.

This paper provides performance analysis of turbo codes over fully interleaved Nakagami- $m$ fading channels with Middleton's additive white Class-A impulsive noise (MAWCAIN). Simulation results for memoryless Nakagami- $m$ fading channels under coherent BPSK signaling are provided for the cases of ideal channel state information (ICSI) and no channel state information (NCSI) at the decoder. As in the 3GPP UMTS forward link an eight state turbo encoder having $(1,13 / 15,13 / 15)$ generator polynomial is used throughout the analysis. The novelty of this work lies in the fact that this is an initial attempt to provide a detailed analysis of turbo codes over Nakagami- $m$ fading channels with impulsive noise rather than fading channels with AWGN.

\section{INTRODUCTION}

In various communication environments the Gaussian noise assumption is insufficient to model the true effect of additive noise due to the presence of more frequent large amplitude excursions from the average value in the signal [1]. This behavior exhibit sharp spikes or occasional bursts of outlying observations than one would expect from the Gaussian distributed signals. Due to this, the density function decay in the tail of such noise sources is less rapid than that of the Gaussian density function. This non-Gaussian noise is prevalent because of either man made noise sources or natural phenomena and can be momentous in many applications and must be taken into consideration to improve system performance. Automobile ignitions, neon lights and many other electronic devices are the common source of man made noise. On the other hand, lightning discharges, impulsive interference in power line channels or in undersea communication systems, noisy made by aquatic animals or surrounding acoustical noises due to ice cracking in arctic regions are some of the inherent means of the occurrence of impulsive noise [1].

In wireless communication environments the performance of communication systems is often degraded by fading in addition to the additive noise. It is often observed that for wireless communication systems in urban areas the additive noise is rather impulsive in nature than Gaussian due to the abundance of man made noise sources. In order to improve the performance of
Erhan AliRiza İnce

Eastern Mediterranean University

Famagusta, North Cyprus

communication systems under such severe conditions forward error correcting codes are used indispensably.

The capability of turbo codes to exhibit excellent performance in AWGN channels close to the channel capacity is provided in detail in [2]-[4]. Similarly, a comprehensive performance analysis of turbo codes via either simulation or analytical means over fading channels is carried out in [5]-[8]. It is well known that the performance of conventional decoders which are designed for AWGN type interference fail to provide good results in impulsive environments [9]. In order to overcome this problem, the decoders must be designed to provide optimized performance in non-Gaussian environments. Recently, authors in $[10,11]$ provided preliminary results for the performance analysis of turbo and LDPC codes over power line channels by modeling the noise component as MAWCAIN [12, 13]. The work presented in [10] and [11] is novel since it is the only known attempt to discuss the performance of turbo and LDPC codes in MAWCAIN environments, despite the existence of an in-depth analysis of optimum or suboptimum receivers for coherent detection in MAWCAIN [14].

This paper extends the analysis of turbo codes to fully interleaved Nakagami- $m$ fading channels with MAWCAIN for the cases of ICSI and NCSI. Extensive simulation results for the Nakagami fading parameter values of $m=1$ (Rayleigh fading case) and $m=3$ (mild fading case) are provided to get an understanding on the performance of turbo codes over the Nakagami- $m$ fading channels under MAWCAIN. During the simulations a rate $1 / 3$ turbo code with a memory of three and input block size of $N$ bits is chosen to generate a frame size of $F S=3(K+3)$ bits as an output stream. For the second constituent encoder a random interleaver is chosen to shuffle the input bit sequence and the first encoder is terminated in the zero state.

The paper is organized as follows: Section I is about the general introduction and literature survey. Section II provides details about the Middleton's additive white Class-A type impulsive noise and the Nakagami- $m$ fading channel. It also reveals the structure of the turbo encoder and decoder which are adopted for performance analysis. Section III focuses on the modified channel reliability expression for both the ICSI and NCSI scenarios in the light of impulsive noise. Section IV provides all the simulation results and a detailed discussion on them. Finally, the findings of this work are summarized in Section $\mathrm{V}$. 


\section{Turbo Decoding OVER NAKAGAMI- $m$ FADing CHANNELS WITH MAWCAIN}

The subsections below give details about the Middleton's additive white Class-A impulsive noise, the Nakagami- $m$ fading distribution followed by the turbo code encoder and decoder structures.

\section{A. Middleton's Class-A Impulsive Noise Model}

Generally, the narrow-band impulsive noise models are established by either modeling the underlying physical mechanism or by using an empirical model [1]. Though, empirical models are mathematically less cumbersome they lack to provide a direct relationship between their parameters and the measurable quantities. Middleton's additive white Class-A impulsive noise model $[12,13]$ is based on the direct characterization of the physical mechanisms that give the noise its impulsive nature. The mathematical representation for this model assumes that the impulsive noise sources are Poisson distributed and always contain the background Gaussian noise. The probability density function (pdf) for MAWCAIN model is

$$
p(x)=\sum_{r=0}^{\infty} \frac{\exp (-A) A^{r}}{r ! \sqrt{2 \pi} \sigma_{r}} \exp \left(\frac{-x^{2}}{2 \sigma_{r}^{2}}\right)
$$

where $r$ is the number of impulsive noise sources, $A$ is called the impulsive index and $\sigma_{r}^{2}$ is defined as

$$
\sigma_{r}^{2}=\frac{\sigma^{2}(r+A \Gamma)}{A(1+\Gamma)}
$$

$\Gamma$ denotes the Gaussian to Impulsive noise power Ratio (GIR) and equals $\sigma_{G}^{2} / \sigma_{I}^{2}$. Furthermore $\sigma_{T}^{2}$ denotes the total noise power and equals

$$
\sigma^{2}=\sigma_{G}^{2}+\sigma_{I}^{2}
$$

At any time instant the noise at the receiver can be characterized by a Gaussian pdf having a variance of

$$
\sigma_{r}^{2}=\sigma_{G}^{2}+\frac{r}{A} \sigma_{I}^{2}=\left(\frac{r+A \Gamma}{A \Gamma}\right) \sigma_{G}^{2} .
$$

For large values of $A(A \geq 10)$, the Class-A impulsive noise becomes continuous and its statistical features become similar to that of the Gaussian noise. Therefore for large values of $A$ Class-A impulsive noise can be modeled as a Gaussian channel.

\section{B. Nakagami-m Fading Distribution}

The Nakagami- $m$ fading model is yet another channel model that can be used to characterize fading environments. The factor $m$ is its shape parameter which controls the severity of amplitude fading [15]. The justification for the use of the Nakagami- $m$ fading model is due to its good fit to empirical data. The normalized Nakagami- $m$ distribution (for $a \geq 0$ ) with its mean and variance is

$$
\begin{gathered}
p(a)=\frac{2 m^{m} a^{2 m-1}}{\Gamma(m)} e^{-m a^{2}}, \quad m \geq \frac{1}{2} \\
m_{a}=\frac{\Gamma\left(m+\frac{1}{2}\right)}{\Gamma(m) \sqrt{m}}
\end{gathered}
$$

$$
\sigma_{a}^{2}=1-\frac{1}{m}\left[\frac{\Gamma\left(m+\frac{1}{2}\right)}{\Gamma(m)}\right]^{2}
$$

where $\Gamma(\cdot)$ is the gamma function. The value $m=1$, correspond to the widely used Rayleigh fading model. Values of $m$ less than unity correspond to fading more severe than Rayleigh whereas, values greater than one represent mild fading.

\section{Turbo Encoder}

This paper utilizes the eight state turbo encoder as depicted in Fig. 1. This encoder was chosen since this structure is used by the $3^{r d}$ generation cellular mobile communication systems based on $3^{\text {rd }}$ Generation Partnership Project (3GPP) [16]. The $3 \mathrm{GPP}$ adopted turbo encoder uses two identical recursive systematic convolutional (RSC) encoders with each constituent encoder having a rate of $1 / 2$. The generator matrix for the turbo encoder is expressed as

$$
G(D):=\left[\begin{array}{ll}
1 & \frac{1+D+D^{3}}{1+D^{2}+D^{3}}
\end{array}\right]
$$

where $D$ represents a unit delay. An alternative representation for the generator polynomials is the representation in octal form $(1,13 / 15,13 / 15)$.

The classical rate $1 / 3$ turbo encoder generates three output sequences. The first one which is referred to as the systematic bits is composed of the information bits $\mathbf{u}=\left(u_{1}, u_{2}, \ldots, u_{N}\right)$. The second output sequence, which corresponds to the first parity bits $\mathbf{p}_{1}=\left(p_{1,1}, p_{1,2}, \ldots, p_{1, N}\right)$ is obtained as a result of encoding the input message sequence $\mathbf{u}$. The third output sequence which provides the second stream of parity bits $\mathbf{p}_{2}=$ $\left(p_{2,1}, p_{2,2}, \ldots, p_{2, N}\right)$ results by encoding the interleaved input message sequence $u$. As a result the turbo encoder is a rate $1 / 3$ block encoder which has $N$ input bits and $F S=3(N+3)$ output bits. The three extra bits in $(N+3)$ are due to the termination of the first encoder to the all zero state.

This work uses BPSK modulation for the transmitted sequence $\mathbf{x}$ (the turbo encoded transmitted sequence). The modulated signal $x \in\{1,-1\}$ and the received bits are represented by $\mathbf{y}$. The AWGN or MAWCAIN noise is assumed to have a variance of $N_{0} / 2$.

\section{Turbo Decoder}

The Turbo decoder uses two component decoders by sharing information to iteratively decode the received sequence $\mathbf{y}$. The decoder is based on the Soft Input/Soft Output (SISO) algorithm which takes as an input a priori information and produces a posteriori information as an output. The BCJR algorithm [17] which is also known as the forward-backward algorithm is the core behind the turbo decoding algorithm. Although, the BCJR algorithm provides optimal results for estimating the outputs of a Markov process in white noise, it suffers form numerical complications due to the use of non-linear functions with mixed multiplication and addition operations. Hence, different derivative of this algorithm such as Log-MAP, Max-Log-MAP or SOVA [18]-[20] are often utilized in practice. This work utilizes the Log-MAP decoding scheme whose 


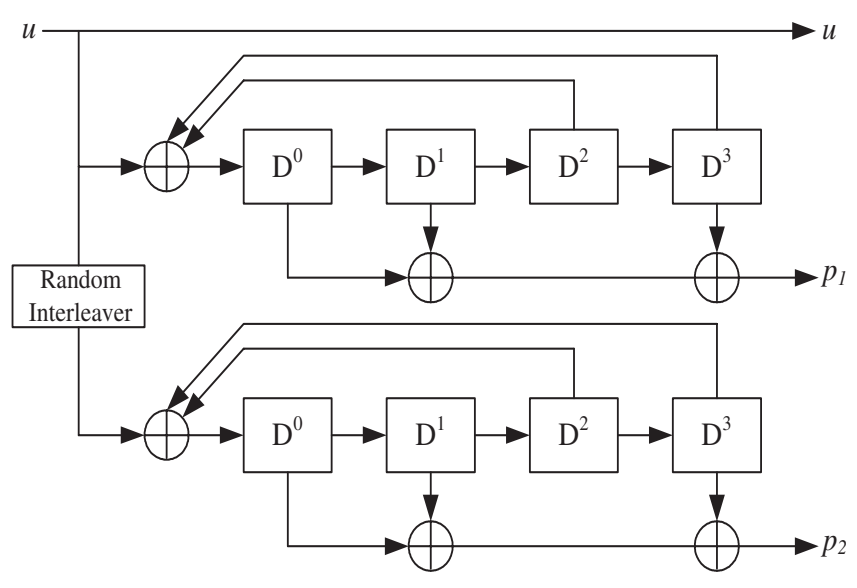

Figure 1: The structure of the rate 1/3 turbo encoder.

details can be found in [20]. The derivation of Log-MAP decoding is based on a-posteriori log-likelihood ratio (LLR) concept, that is, the logarithm of the ratio of the probabilities of a given bit being +1 or -1 given the received (observed) output $\mathbf{y}$. It is well known that the the soft channel output values can be expressed as

$$
L(\hat{\mathbf{x}})=L_{c}(\mathbf{y})+L(\mathbf{x})+L_{e}(\hat{\mathbf{x}})
$$

where $\mathbf{x}$ represents the BPSK modulated sequence whereas $\hat{\mathbf{x}}$ is the estimated soft value. The LLR gives the soft channel output $L(\hat{x})$ in term of three quantities a priori values $L(\mathbf{x})$, extrinsic information $L_{e}(\hat{\mathbf{x}})$ and the channel reliability $L_{c}(\mathbf{y})$ [20]. It was shown in [10] that the performance of turbo decoder in MAWCAIN can be obtained by only adjusting the channel reliability value according to the impulsive noise channel.

\section{Channel Reliability}

The performance of the turbo decoding principle depends on the sharing of information between the constituent decoders. The computation of the LLR gives rise to a variable called the channel reliability [18]. The channel reliability value is also based on the LLR for a particular channel and can be written as follows

$$
L_{c}\left(y_{n}\right)=\ln \left[\frac{P\left(y_{n} \mid x_{n}=1\right)}{P\left(y_{n} \mid x_{n}=-1\right)}\right] .
$$

In order to obtain good turbo code performance over a particular channel the above expression needs to be adopted for the underlying channel. Sub-sections $A$ and $B$ that follow provide the corresponding channel reliability expressions for channels with AWGN or MAWCAIN as the additive noise.

\section{A. Channel Reliability for AWGN}

The channel reliability value for a BPSK modulated data over an AWGN channel with fading can be expressed as

$$
L_{c}\left(y_{n}\right)=\ln \left[\frac{\frac{1}{\sqrt{2 \pi} \sigma} \exp \left(-\frac{\left(y_{n}-a_{n}\right)^{2}}{2 \sigma^{2}}\right)}{\frac{1}{\sqrt{2 \pi} \sigma} \exp \left(-\frac{\left(y_{n}+a_{n}\right)^{2}}{2 \sigma^{2}}\right)}\right]
$$

a simplified version after mathematical manipulations takes the form of

$$
L_{c}\left(y_{n}\right)=4 y_{n} a_{n} \frac{E_{s}}{N_{0}}
$$

In equation (12) $y_{n}$ represents the received bit through the channel whereas $a_{n}$ corresponds to the fading coefficient. In the case of no fading $a_{n}=1$. In the case of fading with no-channel state information the fading value becomes the expected value $a_{n}=E[\mathbf{a}]$ of the underlying fading channel. When we have ideal channel state information available at the decoder then $a_{n}$ takes the exact fading value.

\section{B. Channel Reliability for MAWCAIN}

Similarly, for the MAWCAIN channel model, the channel reliability becomes

$$
L_{c}\left(y_{n}\right)=\ln \left[\frac{\sum_{r=0}^{\infty} \frac{\exp (-A) A^{r}}{r ! \sqrt{2 \pi} \sigma_{r}} \exp \left(-\frac{\left(y_{n}-a_{n}\right)^{2}}{2 \sigma_{r}^{2}}\right)}{\sum_{r=0}^{\infty} \frac{\exp (-A) A^{r}}{r ! \sqrt{2 \pi} \sigma_{r}} \exp \left(-\frac{\left(y_{n}+a_{n}\right)^{2}}{2 \sigma_{r}^{2}}\right)}\right] .
$$

Again, identical interpretation follows as for the case of AWGN channel. Other than this, since the MAWCAIN channel consists of an infinite series the above equation will be too cumbersome to compute. A simple rule as suggested in [10] is to truncate the series for $r=0,1, \ldots, L$ where $L=\max \{2,\lceil 10 A\rceil\}$. A basic interpretation of the above rule is that for small index values of $r$ only the first few terms in the summation are significant due to $e^{-A} A^{r} / r$ !. Hence, higher index values can be ignored. As the value of $A$ is increased the number of significant terms in the summation also increases and hence more terms are need to obtain a better reliability value.

Fig. 2 shows the graphical representation of the channel reliability for various cases of $\Gamma$ and $A$ in MAWCAIN channel when both $\sigma$ and a are unity. Additionally, the channel reliability expression for AWGN channel is also provided in the plot. A simple observation from Fig. 2 is that the LLR values $L_{c}\left(y_{n}\right)$ in MAWCAIN channel has a nonlinear behavior in contrast to the Gaussian channel case. The channel reliability value at $y_{n}=+1$ for MAWCAIN channel is higher than the reliability value for the AWGN channel. Smaller values of $\Gamma$ when $A$ is constrained gives rise to a peak around $y_{n}=1$. On the other hand changing values for $\mathrm{A}$ when $\Gamma$ is constrained shifts the local minimum in the vicinity of $y_{n}=+2$ and causes a wider spread for smaller $A$ values. It is worth noting that smaller values for $A$ correspond to more impulsive channels whereas, smaller values for $\Gamma$ point to the fact that power in the impulsive noise component is greater than the power in the Gaussian. From these observation one expects the turbo decoder to provide better performance when both $A$ and $\Gamma$ take small values.

For the practical use of turbo decoding over MAWCAIN channels, one needs to estimate the impulsive index $A$, the Gaussian to impulsive noise power ratio $\Gamma$, and the noise power $\sigma^{2}$. These parameters can be obtained through the second, fourth and sixth moments of the received envelopes [21]. Sim- 


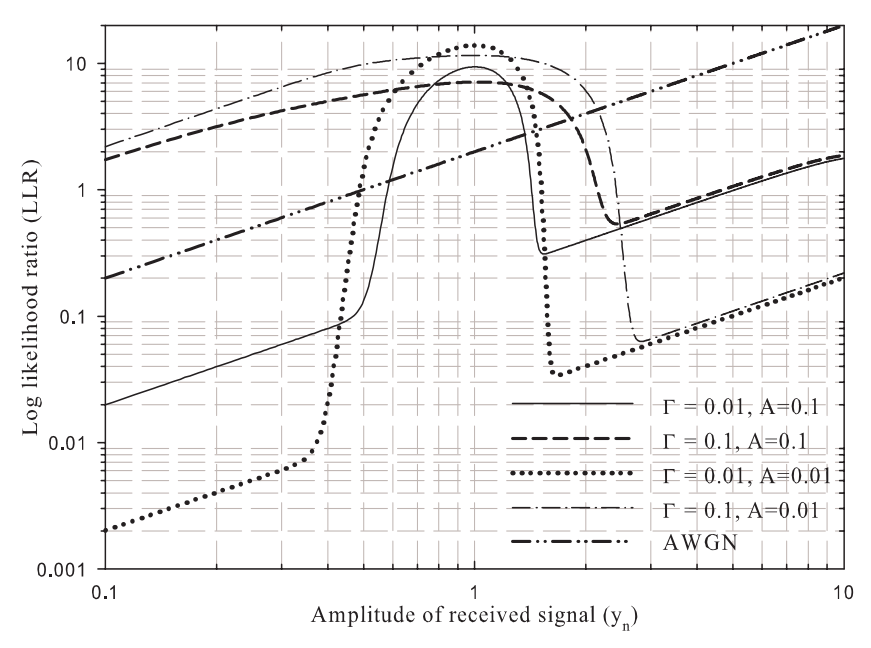

Figure 2: LLR for AWGN and MAWCAIN channels with both $\sigma$ and a equal to unity for various values of $\Gamma$ and $A$.

ilarly, the fading statistics needs to be obtained using estimation techniques according to the decoder type which either uses ICSI (requires the knowledge of the fading coefficients) or NCSI (requires the knowledge of the mean value of the fading coefficients).

\section{RESUlts AND Discussion}

Fig. 3 illustrate the simulation results of turbo codes over Nakagami- $m$ fading channels under ICSI with MAWCAIN by using an input message block length of $N=5000$ bits and five decoding iterations. For the analysis two values of Nakagami fading parameter $m=1$ and $m=3$ are chosen to see the performance under severe and mild fading conditions. It can be seen from Fig. 3 that when $A=0.01$ (i.e. more impulsive channel) there does not exist any noticeable performance difference for $\Gamma=0.1$ and $\Gamma=0.01$ when the channel is either mild $(m=3)$ or severe $(m=1)$. Contrary to this, when $A=0.1$ (i.e. less impulsive channel than $A=0.01$ ) smaller value of $\Gamma=0.01$ provides better performance than the higher value of $\Gamma=0.1$. This is due to the reason that the proposed turbo decoder suppresses more impulsive noise power. Similarly, Fig. 4 provides the simulation results of turbo codes over Nakagami- $m$ fading channels under NCSI with MAWCAIN by using an input message block length of $N=5000$ bits and five decoding iterations. Again the two different values of Nakagami- $m$ fading parameter $m=1$ and $m=3$ are chosen to see the performance under severe and mild fading conditions. From Fig. 4 one can easily notice that when $A=0.01$ (i.e. more impulsive channel) there does not exist any noticeable performance difference for $\Gamma=0.1$ and $\Gamma=0.01$ when the channel is either mild ( $m=3$ ) or severe $(m=0)$. Contrary to this, when $A=0.1$ (i.e. less impulsive channel than $A=0.01$ ) smaller value of $\Gamma=0.01$ provides better performance than the higher value of $\Gamma=0.1$.

It is obvious from Fig. 3 and Fig. 4 that the turbo decoder for ICSI perform better than NCSI case, which is expected due to

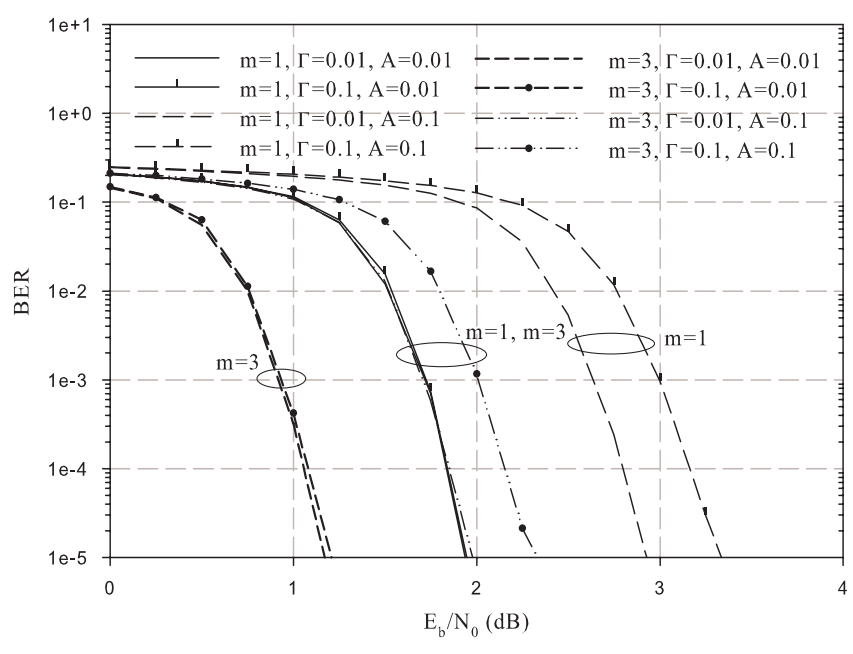

Figure 3: Simulated BER performance of $(1,13 / 15,13 / 15)$ turbo code over Nakagami- $m$ fading channels under ICSI with MAWCAIN for a message block length of $N=5000$ bits after 5 decoding iterations.

the availability of exact channel fading coefficients. It is also worth noticing that for mild fading scenario $(m=3)$ the ICSI performs approximately $0.4 \mathrm{~dB}$ better than NCSI at a BER of $10^{-5}$ whereas, the difference between the two for severe fading case $(m=1)$ is around $1 \mathrm{~dB}$ (for $\Gamma=0.01$ and $A=0.01$ ) again at a BER of $10^{-5}$. The performance difference between ICSI and NCSI is less when the fading conditions are mild due to the fact that as the fading becomes mild, the multiplicative effect tends towards unity and the performance of ICSI and NCSI decoders become similar.

\section{CONCLUSION AND REMARKS}

This article discusses the performance analysis of turbo codes over fully interleaved Nakagami- $m$ fading channels under Middleton's additive white Class-A impulsive noise. The work provides the updated channel reliability expression along with its interpretation for the impulsive channel. A detailed performance analysis is provided for the ideal-CSI and no-CSI cases. The work presented herein is novel in a sense that according to authors' best knowledge it is a first attempt of its kind to provide performance analysis of turbo codes in Nakagami- $m$ fading channels under MAWCAIN.

\section{REFERENCES}

[1] K.S. Vestola, "Threshold Detection in Narrow-Band Non-Gaussian Noise", IEEE Transcations on Communications, Vol. COM-32, No. 2, February 1984.

[2] C. Berrou, A. Glavieux, and P. Thitimajshima, Near Shannon Limit Error-Correcting Coding and Decoding: Turbo Codes, in Proceedings of the IEEE International Conference on Communications (ICC), pp. 1064-1070, 1993.

[3] D. Divsalar, S. Dolinar, R. McEliece, and F. Pollar, Transfer Function Bounds on the Performance of Turbo Codes, JPL TDA Progress Report, Vol. 42, pp. 44-55, August 1995. 


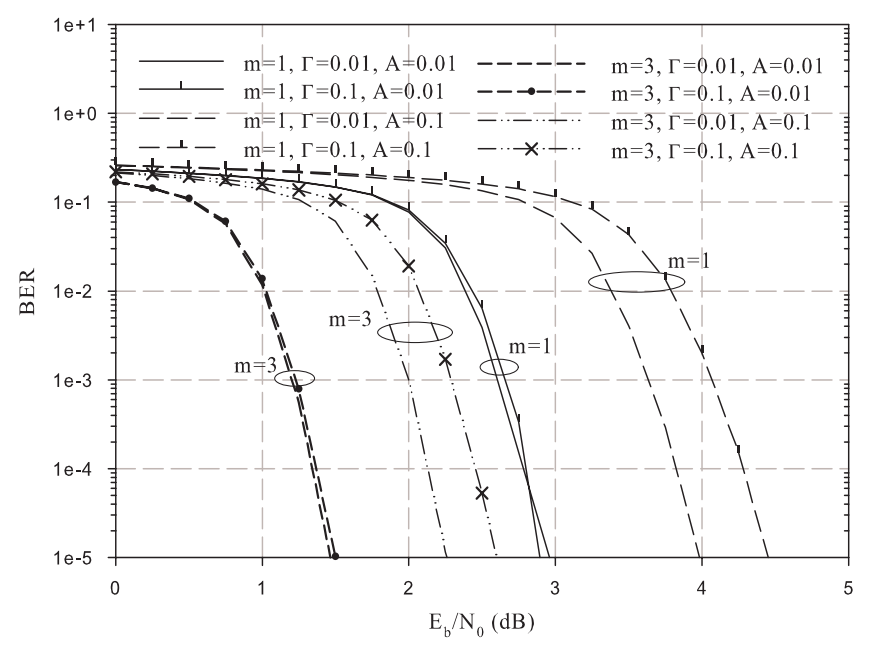

Figure 4: Simulated BER performance of $(1,13 / 15,13 / 15)$ turbo code over Nakagami- $m$ fading channels under NCSI with MAWCAIN for a message block length of $N=5000$ bits after 5 decoding iterations.

[4] S. Benedetto and G. Montorsi, "Unveiling Turbo Codes: Some Results on Parallel Concatenated Coding Schemes", IEEE Transactions on Information Theory, Vol. 42, pp. 409-429, March 1996.

[5] E.K. Hall and S. Wilson, "Design and Analysis of Turbo Codes on Rayleigh Fading Channels", IEEE Journal on Selected Areas in Communications, pp. 160-174, February 1998.

[6] S.A. Ali, N.S. Kambo and E.A. İnce, "Exact Expression and tight Bound on Pairwise Error Probability for Performance Analysis of Turbo Codes Over Nakagami- $m$ Fading Channels", IEEE Communications Letters, Vol. 11, No. 5, pp. 399-401, May 2007.

[7] S.A. Ali and E.A. İnce, A Simple Moment Based Approximation to Pairwise Error Probability for Memoryless Fading Channels, in Proceeding of the IEEE $16^{\text {th }}$ International Symposium on Personal Indoor and Mobile Radio Communication (PIMRC), pp. 2543-2546, September 2005.

[8] I. Sason and S. Shamai (Shitz), "On Improved Bounds on the Decoding Error Probability of Block Codes Over Interleaved Fading Channels, with Applications to Turbo-Like Codes", IEEE Transactions on Information Theory, Vol. 47, No. 6, pp. 2275-2299, September 2001.

[9] C. Tepedelenlioğlu and P. Gao, "On Diversity Reception Over Fading Channels With Impulsive Noise", IEEE Transactions on Vehicular Technolgy, Vol. 54, No. 6, pp. 3676-3680, November 2005.

[10] D. Umehara, H. Yamaguchi and Y. Morihiro, Turbo Decoding in impulsive noise environment, in Proceedings of the Globecom 2004, Dallas, Texas, USA, pp. 194-198, November/December 2004.

[11] H. Nakagawa, D. Umehara, S. Denno and Y. Morihiro, A Decoding for Low Density Parity Check Codes over Impulsive Noise Channels, in Proceedings of the International Symposium on Power Line Comm. and its Applications, pp. 85-89, 2005.

[12] D. Middleton, "Statistical-physical model of electromagnetic interference", IEEE Transactions on Elect. Comp., Vol. EMC-19, No. 3, pp. 106-126, 1977.

[13] D. Middleton, "Non-Gaussian noise models in signal processing for telecommunications: new methods and results for class A and class B noise models", IEEE Transactions on Information Theory, vol. 45, No. 4, pp. 1129-1149, May 1999.

[14] A.D. Spaulding and D. Middleton, "Optimum reception in an Impulsive Interference Environment-Part I: Coherent Detection", IEEE Transactions on Communications, Vol. COM-25, No. 9, pp. 910-923, 1977.

[15] M. Nakagami, The $m$-distribution, a General Formula of Intensity Distribution of Rapid Fading, Statistical Methods in Radio Wave Propagation, W. G. Hoffman, Ed, Oxford, England: Pergamon, 1960.
[16] $3^{\text {rd }}$ Generation Partnership Project (3GPP) Technical Specification Group: Radio Access Network Working Group 1, Multiplexing and Channel Coding, TS 25. 212 v1.1.0, June 1999.

[17] L.R. Bahl, J. Cocke, F. Jelinek and J. Raviv, "Optimal Decoding of Linear Codes for Minimizing Symbol error Rate", IEEE Transactions on Information Theory, IT-20, pp. 284-287, March 1974.

[18] J. Hagenauer and P. Robertson, Iterative Turbo Decoding of Systematic Convolutional Codes with MAP and SOVA algorithms, in Proceedings of the ITG Conference on Source and Channel Coding, October 1994.

[19] J. Hagenauer, E. Offer and L. Papke, "Iterative Decoding of Binary Block and Convolutional Codes", IEEE Transactions on Information Theory, Vol. 42, No.2, March 1996.

[20] B. Vucetic and J. Yuan, Turbo Codes: Principles and Applications, Kluwer Academic Publishers, Boston 2000.

[21] D. Middleton, "Procedures for determining the parameters of the first order canonical models of Class A and Class B electromagnetic interference", IEEE Transactions on Electromagn. Compact., Vol. EMC-21, No. 3, pp. 190-208, August 1979. 